Collection: COST Action FP0903 (2010) - Rome (Italy)

"Research, monitoring and modelling in the study of climate change and air pollution impacts on forest ecosystems"

Guest Editors: E Paoletti, J-P Tuovinen, N Clarke, G Matteucci, R Matyssek, G Wieser, R

Fischer, P Cudlin, N Potocic

\title{
COST Action FP0903: “Research, monitoring and modelling in the study of climate change and air pollution impacts on forest ecosystems"
}

\author{
Paoletti $\mathrm{E}^{(1)}$, Tuovinen J-P(2)
}

The COST Action FP0903 and its first scientific conference are described here. This Action aims at increasing the understanding of state and potential of forest mitigation and adaptation to climate change in a polluted environment. Another key objective is to reconcile process-oriented research, long-term monitoring and applied modelling, for which the concept of "Supersites" (comprehensive forest research sites) is being developed. The conference "Research, monitoring and modelling in the study of climate change and air pollution impacts on forest ecosystems" was held in Rome (Italy) on 5-7 October 2010. It networked the main European communities working on air pollution, climate change and forests. The proceedings of the conference are published in this collection.

Keywords: Air Pollution, Climate Change, Forests, COST

\section{Introduction to COST and Action FP0903}

COST - the acronym for European Cooperation in Science and Technology - is the oldest and widest European intergovernmental network for cooperation in research (http://www.cost.eu). Established by the Ministerial Conference in November 1971, COST is presently used by the scientific communities of 36 European countries to cooperate in common research projects supported by national funds. The funds provided by COST support cooperation networks, orga-

(1) IPP-CNR, v. Madonna del Piano 10, I50019 Sesto Fiorentino (Florence - Italy); (2) Finnish Meteorological Institute, P.O. Box 503, FI-00101 Helsinki (Finland)

\section{(a) Elena Paoletti (e.paoletti@ipp.cnr.it)}

Received: Jun 29, 2011 - Accepted: Jul 14, 2011

Citation: Paoletti E, Tuovinen J-P, 2011. COST Action FP0903: "Research, monitoring and modelling in the study of climate change and air pollution impacts on forest ecosystems". iForest 4: 160-161 [online 2011-08-11] URL:

http://www.sisef.it/iforest/ show.php? id $=583$ nized as COST Actions, through which more than 30000 European scientists are involved in research. A "bottom-up approach" (the initiative of launching a COST Action comes from the European scientists themselves), "à la carte participation" (only countries interested in the Action participate), "equality of access" (participation is open also to the scientific communities of countries not belonging to the European Union) and "flexible structure" (easy implementation and light management of the research initiatives) are the main characteristics of COST. As precursor of advanced multidisciplinary research, COST has a very important role in the realisation of the European Research Area (ERA), anticipating and complementing the activities of the Framework Programmes, constituting a "bridge" towards the scientific communities of emerging countries, increasing the mobility of researchers across Europe and fostering the establishment of "Networks of Excellence" in many key scientific domains.

Action FP0903 "Climate Change and Forest Mitigation and Adaptation in a Polluted Environment - MAFor" (http://costfp0903.ipp.cnr.it/) creates a platform of experts from different fields, with the following main objectives: (1) to increase understanding of state and potential of forest miti- gation and adaptation to climate change in a polluted environment; and (2) to reconcile process-oriented research, long-term monitoring and applied modelling at comprehensive forest research sites (so-called Supersites).

The secondary aims of MAFor are:

1. to expand the evaluation of the presently available data;

2. to identify current knowledge gaps and emerging research needs;

3. to optimise the geographical distribution of Supersites, ensuring the coverage of the most relevant ecosystems in Europe;

4. to assess the value and ecological meaning of measurements and identify which measurements are a priority;

5. to develop criteria and standardized protocols for data acquisition, processing, upscaling and storage;

6. to promote and facilitate the use of the results beyond the initially targeted research communities.

In particular, MAFor aims at translating the existing European knowledge on climate and air pollution dynamics into impacts on forests, with a focus on carbon sequestration, water and nutrient cycles, responses to nitrogen deposition and ozone exposure. Interactions of different stressors and the effects on forest services are still poorly understood under real-world conditions, mostly because of limited interaction between the disciplinary-oriented scientific communities. Present efforts are in fact highly fragmented. The existing national and European research, monitoring and modelling communities work separately and often do not have the opportunity to exchange data and concepts. Networking those communities is a key rationale of this Action. A summary of the main networks/institutions MAFor collaborates with is shown in Fig. 1.

\section{The kick-off conference of Action FP0903}

The kick-off conference of the COST Action FP0903 was held in Rome, Italy on 5-7 October, 2010. It was attended by 82 participants from 25 countries including Canada and Japan. Details are available at http://cost-fp0903.ipp.cnr.it/events/cost-action-fp0903-first-conference.html. A total of 47 full presentations and 10 short talks were given. Of these, 30 presentations were about monitoring, 14 dealt with experimental research, nine were about modelling and the remaining four addressed the integration of research and monitoring. The dominant subjects were the carbon balance $(23 \%)$, the nitrogen cycle $(16 \%)$, climate change (14\%) and ozone pollution $(12 \%)$. A final round table discussed the concept and design of Supersites, at which comprehensive plant, soil and atmospheric research and monito- 
Fig. 1 - Main subjects and interactions of the COST Action FP0903. Concerning ozone, the main target groups are EMEP and ICP Forests.

About nitrogen, the main programme is NitroEurope. The COST Action ES0804 networks greenhouse gas measurements of NitroEurope and other carbon-fluxes programmes. Meteorological data are available by several programmes across Europe, but a few work at forest sites; they are linked to some of the above-mentioned programmes. A number of impacts on forests are monitored at ICP

Forests sites and ICP Integrated Monitoring sites, which both are part of the LTER programme.

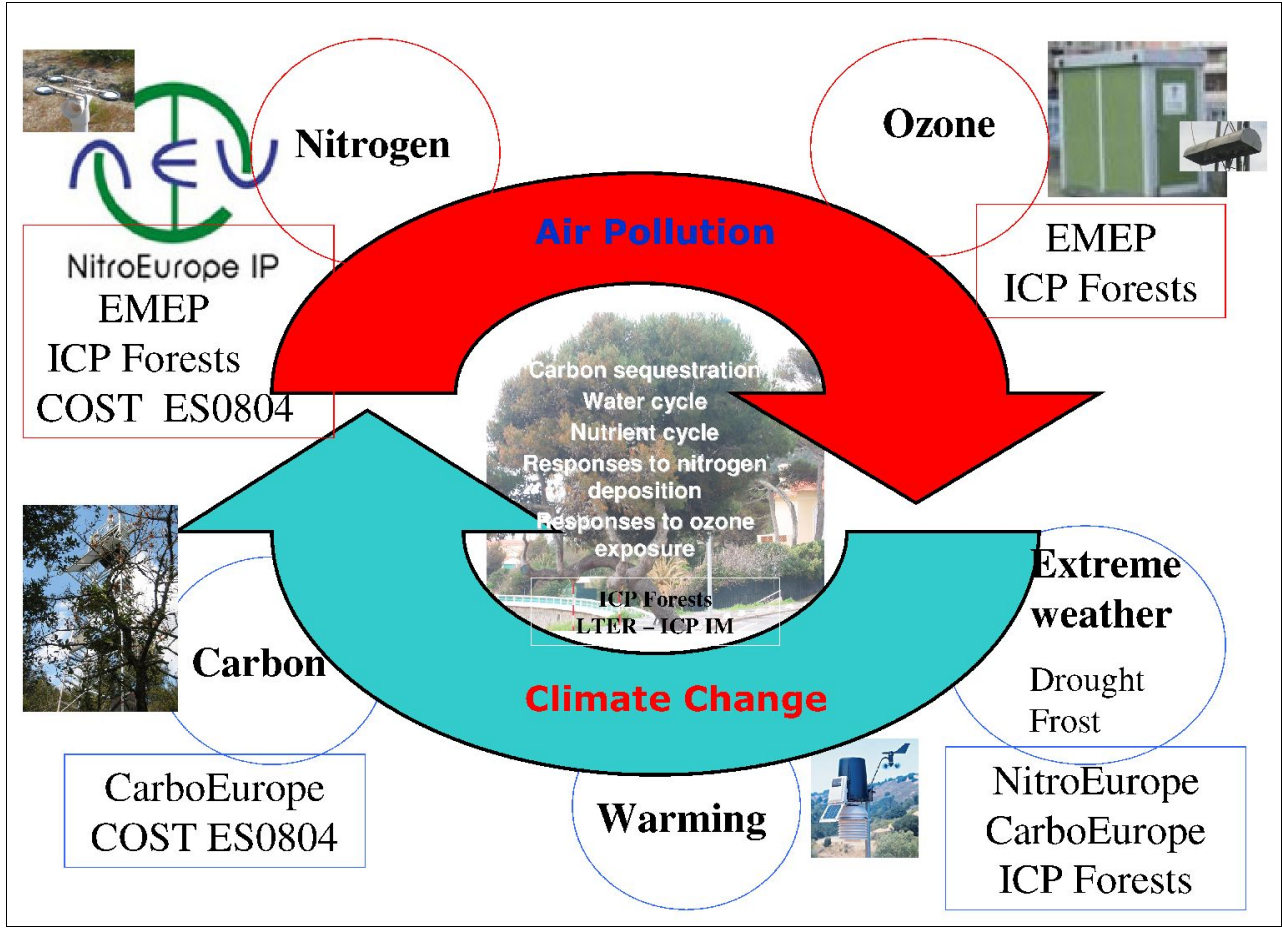

ring can be carried out. The discussion was attended by the following representatives of top-level European networks: David Leaver and Wim De Vries (NitroEurope), Lars Lundin (ICP IM), Dario Papale (CarboEurope), David Simpson (EMEP), Timo Vesala (ICOS), Michael Mirtl (LTER), JuhaPekka Tuovinen (COST FP0903) and Richard Fischer (ICP Forests).

The conference networked different communities working with air pollution, climate change and forests in Europe. Common problems were indentified and discussed, including data availability and accessibility, topical research, monitoring and modelling prospects, and the need and interest for joint Supersites. In addition, possibilities for fur- ther developments were analysed focusing on the merging of different databases, refinement of research hypotheses and modelling approaches, and streamlining of common methodologies. The proceedings of the conference are published in this special issue.

The conference and these proceedings are dedicated to the memory of John Derome who passed away on June 7, 2010. John was a highly reputed soil scientist specialized in nutrient budgets and air pollution effects. $\mathrm{He}$ was a member of the Management Committee of this Action for Finland and gave a great contribution of experience and advice to the progress of the Action. We are missing him, in particular his proactivity, sense of humour and warm personal charisma.

\section{Acknowledgements}

Joint publication of the COST Action FP0903 Climate Change and Forest Mitigation and Adaptation in a Polluted Environment. We thank: the colleagues of the scientific committee for selecting the speakers and contributing to the programme; Melae Langbein from the COST office for her continuous support to the Action; the National Council of Research of Italy for hosting the conference in Rome; the organising secretary Clementina Falco for her enthusiastic support; and the speakers and attendees, for the stimulating presentations and discussion. 